\title{
G-band spectral synthesis and diagnostics of simulated solar magneto-convection
}

\author{
S. Shelyag ${ }^{1}$, M. Schüssler ${ }^{1}$, S. K. Solanki ${ }^{1}$, S. V. Berdyugina ${ }^{2,3}$, and A. Vögler ${ }^{1}$ \\ 1 Max-Planck-Institut für Sonnensystemforschung ${ }^{\star}, 37191$ Katlenburg-Lindau, Germany \\ e-mail: shelyag@linmpi.mpg.de \\ 2 Institut für Astronomie, ETH-Zentrum, 8092 Zürich, Switzerland \\ 3 Astronomy Division, PO Box 3000, 90014 University of Oulu, Finland \\ Received 18 March 2004 / Accepted 5 July 2004
}

\begin{abstract}
Realistic simulations of radiative magneto-convection in the solar (sub)photosphere are used for a spectral synthesis of Fraunhofer's $G$ band, which is dominated by spectral lines from the $\mathrm{CH}$ molecule. It is found that the spatial pattern of integrated $G$-band brightness closely matches the spatial structure of magnetic flux concentrations in the convective downflow regions. The brightness contrast is mainly caused by the weakening of $\mathrm{CH}$ lines due to the reduced $\mathrm{CH}$ abundance and the resulting shift of the optical depth scale in the hot and tenuous magnetic flux concentrations. Various properties of the synthetic brightness images agree well with $G$-band observations. These results lends credit to the observational usage of $G$-band bright features as proxies for magnetic flux concentrations in the solar photosphere. However, the converse is only correct in a limited sense: only a fraction of the magnetic flux concentrations turn out to be bright in the $G$ band.
\end{abstract}

Key words. Sun: magnetic fields - Sun: photosphere

\section{Introduction}

The interaction between magnetic field and convective motion in the solar (sub)photosphere results in the formation of small-scale concentrations of magnetic flux (Spruit et al. 1991; Solanki 1993), which play an important role for the heating of the upper layers of the solar atmosphere and for the variability of total and spectral radiance (Parker 1983; Schrijver et al. 1998; Spruit 2000; Fligge et al. 2000; Krivova et al. 2003). Direct studies of the magnetic field and its dynamics on these small-scale $(\sim 100 \mathrm{~km})$ magnetic elements through Zeeman polarimetry is hampered owing to the seeing effects of the turbulent terrestrial atmosphere.

Images of the solar surface layers taken in Fraunhofer's $G$ band, a spectral region in the wavelength range 429.5-431.5 nm, which is populated by many spectral lines of the $\mathrm{CH}$ molecule, show bright features in the intergranular downflow regions, the so-called $G$-band bright points (Muller \& Roudier 1984; Muller 1985; Berger \& Title 1996; Rutten et al. 2001; Langhans et al. 2002). The $G$-band bright points are believed to correspond to the locations of the magnetic flux concentrations and thus are often used as a "proxies" for small-scale magnetic elements (Berger et al. 1995, 1998; van Ballegooijen et al. 1998; De Pontieu 2002).

In this paper, we use results from 3D radiative MHD simulations (Vögler 2003; Vögler \& Schüssler 2003; Vögler et al. 2004) and spectral line synthesis (Berdyugina et al. 2003) to provide a theoretical basis for such "proxy magnetometry".

\footnotetext{
^ Formerly Max-Planck-Institut für Aeronomie
}

We consider simulations with two different average magnetic field strengths (200 $\mathrm{G}$ and $10 \mathrm{G}$ ), which we take as representing magnetically active and "quiet" solar regions, respectively. In order to study the physical mechanism responsible for $G$ band brightening in magnetic flux concentrations, we calculate synthetic $G$-band images from the MHD simulation results. These images are then compared with observations after proper smoothing to account for image deterioration induced by the telescope and the terrestrial atmosphere. Some results for the $200 \mathrm{G}$ run have been already reported by Schüssler et al. (2003). The correlation of the $\mathrm{CH}$ molecule abundance with the thermodynamical quantities in the magnetic elements shows that a decreased $\mathrm{CH}$ abundance leads to weakening of the $\mathrm{CH}$ lines and thus causes the $G$-band brightening of the magnetic flux concentrations. We find a strong positive correlation between $G$-band brightness and magnetic field strength in the downflow regions.

The paper is organized as follows: in Sect. 2, the MHD simulations are briefly described. Section 3 describes the spectral synthesis code and simulations of the $G$-band spectrum. The mechanism of $G$-band brightening associated with magnetic flux concentrations is discussed in Sect. 4. Section 5 gives a comparison of the simulation with observational data. Conclusions are provided in Sect. 6.

\section{Radiative MHD simulations}

Realistic solar magneto-convection simulations aim at representing the radiative and magnetohydrodynamical processes in 

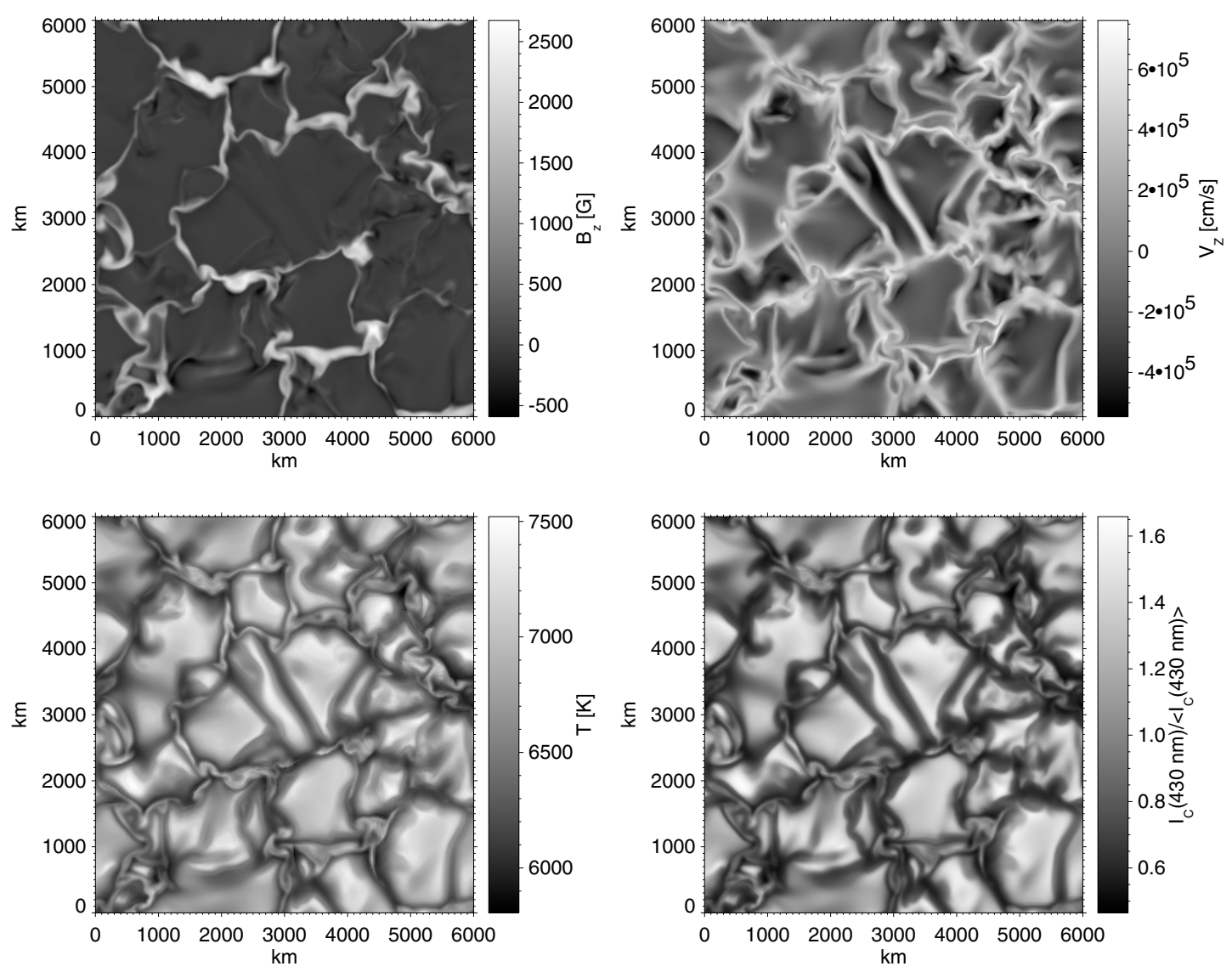

Fig. 1. Maps of physical quantities for a snapshot from the run with $200 \mathrm{G}$ average vertical magnetic field. Upper left: vertical component of the magnetic field at the level $\tau=1$ at $500 \mathrm{~nm}$, corresponding to the visible solar surface. The image shows magnetic flux concentrations in the intergranular lanes. Upper right: vertical component of the velocity at the level $\tau=1$ at $500 \mathrm{~nm}$. Positive (negative) values correspond to downflows (upflows). Lower left: gas temperature on the level $\tau=1$ at $500 \mathrm{~nm}$. There are local enhancements of the temperature in the regions of strong magnetic field in intergranular lanes. Lower right: normalized continuum intensity at $430 \mathrm{~nm}$. The image shows brightenings in the magnetic flux concentrations, which closely correspond to the local temperature enhancements and magnetic flux concentrations.

the solar photosphere and the uppermost layers of the convection zone, so that the results can be directly compared with the observations. We have used the MURaM $^{1}$ code, a 3D MHD code including non-grey radiative transfer and the effects of partial ionization for the 11 most abundant chemical elements (Vögler et al. 2003, 2004).

The size of the computational domain for the simulations considered here is $6000 \times 6000 \times 1400 \mathrm{~km}^{3}$ with a resolution $288 \times 288 \times 100$ grid points. The domain has periodic side boundaries, a closed top and an open bottom boundary. The simulation starts with a plane-parallel atmosphere extending between $800 \mathrm{~km}$ below and $600 \mathrm{~km}$ above the level of optical depth unity at $500 \mathrm{~nm}$. After convection has developed and both the outgoing energy flux and the total kinetic energy have reached stationary values (with only short-term fluctuations on the granulation time scale), a homogeneous vertical magnetic field is introduced. We consider snapshots from two runs with different initial magnetic field strengths of $10 \mathrm{G}$ and $200 \mathrm{G}$

1 The MURaM (MPS/University of Chicago Radiative MHD) code has been developed by the MHD simulation groups at the Max-Planck-Institut für Sonnensystemforschung Katlenburg-Lindau (A. Vögler, S. Shelyag, M. Schüssler) and at the University of Chicago (F. Cattaneo, Th. Emonet, T. Linde). corresponding to "quiet" and active solar regions, respectively. Within a few minutes of simulated time, most of the magnetic flux is assembled in the downflow regions of the convection pattern. After the decay of all transients caused by the introduction of the magnetic field, we have continued the simulations for about another hour of solar time to be sure that the results have become independent of the initial distribution of magnetic flux. Some quantities from the $200 \mathrm{G}$ simulation snapshot are shown in Fig. 1. The snapshot is taken about $160 \mathrm{~min}$ after the start of simulation and 95 min after the vertical magnetic field was introduced.

The lower-left part of Fig. 1 shows the temperature field at the continuum optical depth level $\tau=1$ at $500 \mathrm{~nm}$. The magnetic field map in the upper-left panel shows concentrations of magnetic flux up to $2700 \mathrm{G}$ field strength, which are mainly located in intergranular downflow regions as evident from the map of the vertical velocity component shown on the upperright panel of Fig. 1. Most magnetic flux concentrations in the intergranular lanes correspond to local temperature enhancements (at equal optical depth) caused by partial evacuation and radiative heating of the flux concentrations by hot surrounding plasma (Spruit 1976; Deinzer et al. 1984; Knölker et al. 1991; Vögler \& Schüssler 2003). 

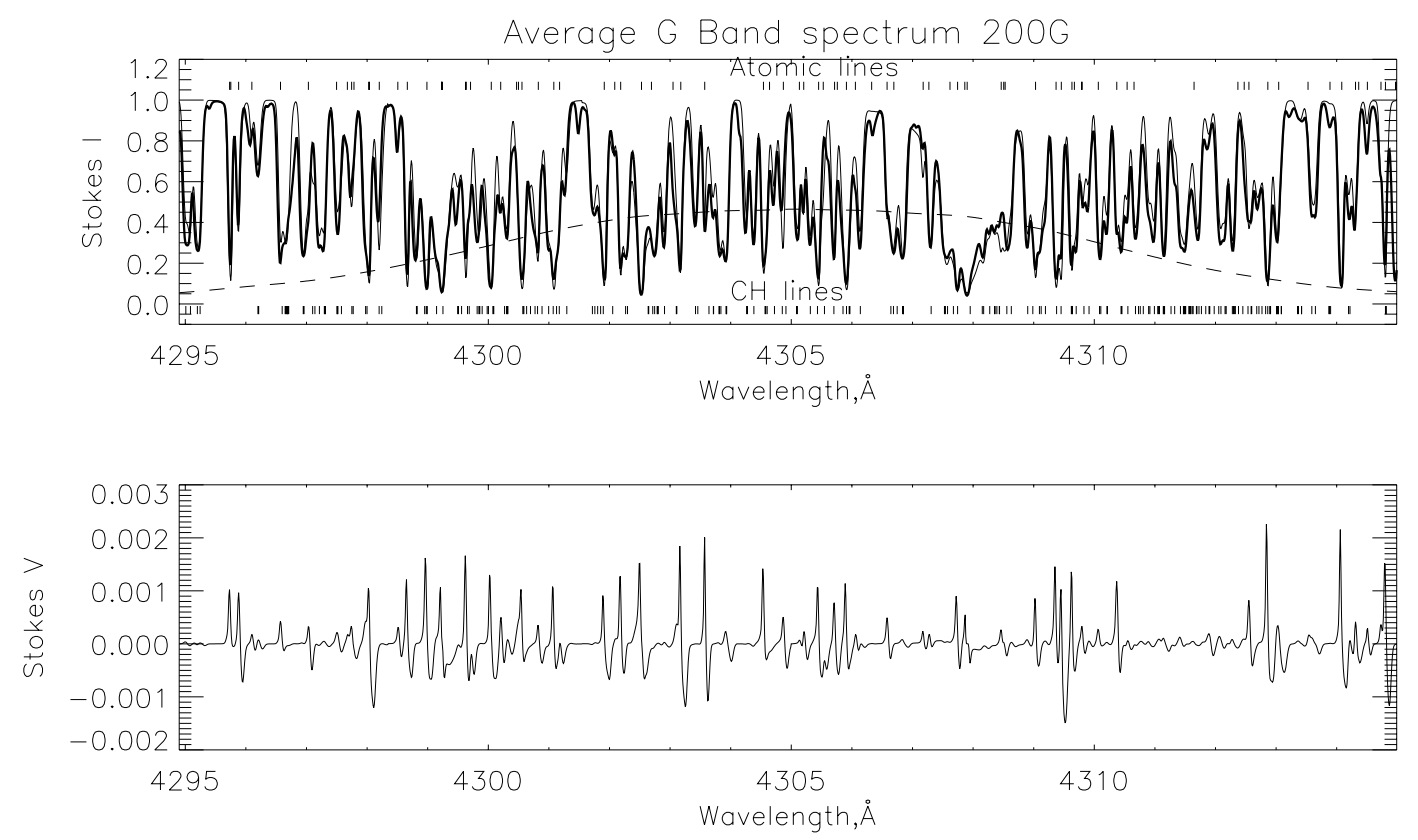

Fig. 2. Spatially averaged spectra of Stokes $I$ (top) and Stokes $V$ (bottom) calculated for a snapshot from the run with $10 \mathrm{G}$ average vertical magnetic field (thin lines). Atomic and molecular lines are indicated on the Stokes- $I$ plot. The dashed line shows the shape of the filter function which is used for calculating the $G$-band intensity. The thick line in the upper panel shows the observed spectrum of the quiet Sun (Delbouille et al. 1973).

\section{Molecular abundances and spectral synthesis}

We have used the STOPRO (STOkes PROfiles) code developed by C. Frutiger, S. K. Solanki and S. Berdyugina (Berdyugina et al. 2003) to calculate the Stokes parameters for atomic and molecular spectral lines in local thermodynamic equilibrium (LTE). In the case of $\mathrm{CH}$ lines, LTE is generally considered valid since the photodissociation of the $\mathrm{CH}$ molecule through bound levels of excited states is negligible in the solar atmosphere (Sánchez Almeida et al. 2001), the ionization energy of carbon is high and it remains primarily neutral in the solar atmosphere, so there are no effects of overionization as they may occur for molecules with metal compounds. Furthermore, there is no scattering polarization observed in $\mathrm{CH}$ lines within the $G$ band at the solar limb in quiet regions, indicating that the line formation is controlled by collisions rather than radiative processes (Gandorfer 2002).

The molecular and atomic line data table which has been used for our $G$-band calculations consists of $241 \mathrm{CH}$ lines and 87 atomic lines, mainly from neutral iron. The $G$-band spectrum emerging along vertical rays is calculated for each of the $288 \times 288$ pixels corresponding to the simulation grid. The calculated $G$-band spectra have 2055 frequency points covering the wavelength range from $429.5 \mathrm{~nm}$ to $431.5 \mathrm{~nm}$. In order to obtain the $G$-band brightness, we multiply the spectra by a filter function centered at $430.5 \mathrm{~nm}$ with $F W H M$ of $1.2 \mathrm{~nm}$, similar to the characteristics of filters used for observations, and integrate over the $G$-band wavelength range.

The spatially averaged $G$-band spectrum (Stokes $I$ and $V$ ) calculated for a snapshot from the run with $10 \mathrm{G}$ average vertical field is shown in Fig. 2. The dashed line indicates the filter function used for calculating the integrated $G$-band intensity. The thick solid line shows the average observed spectrum of quiet Sun (Delbouille et al. 1973). Atomic and $\mathrm{CH}$ lines are marked by small vertical dashes at the top and bottom of the Stokes- $I$ plot, respectively. Whereas the Stokes- $I$ spectrum is strongly affected by the molecular band, the Stokes- $V$ spectrum is dominated by atomic lines, which are more sensitive to the magnetic field. The effective Landé factors of the $\mathrm{CH}$ lines are mostly of the order of 0.1-0.2 (Berdyugina \& Solanki 2002) ${ }^{2}$. There is no strong response of the molecular lines to the magnetic field, so that we do not have a direct influence of the magnetic field on the integrated $G$-band intensity.

Figure 3 shows the $G$-band brightness distributions (upper panels) together with maps of the absolute field strength (lower panels) for the two simulation snapshots of quiet and active regions, corresponding to $10 \mathrm{G}$ (right panels) and $200 \mathrm{G}$ (left panels) average vertical magnetic field, respectively. One can see strong local brightenings up to $I_{G} /\left\langle I_{G}\right\rangle=2.2$ corresponding to the regions of concentrated magnetic field. On the corresponding continuum intensity image (Fig. 1) the same brightenings have a relative brightness of about 1.7 . The close visual correspondence of bright structures in the $G$ band and strong magnetic field regions in both cases is not so clear in the scatter plots of $G$-band brightness vs. magnetic field strength shown in Fig. 4. For $B>500 \mathrm{G}$, the $G$-band brightness indeed increases rapidly with $B$ (a roughly quadratic dependence seems indicated). However, there are also magnetic features with negative $G$-band contrast $\left(I_{G} /\left\langle I_{G}\right\rangle<1\right)$. In the $200-G$ run, this applies to about $30 \%$ of the points with $B>1000 \mathrm{G}$ field. In most cases, these points correspond to the peripheral parts of the expanding magnetic flux concentrations, where the line of sight

\footnotetext{
2 The effective Landé factors in this paper are a factor of 2 too large (E. Landi degl'Innocenti, private communication). The correct values have been used for Stokes profile calculations in STOPRO.
} 

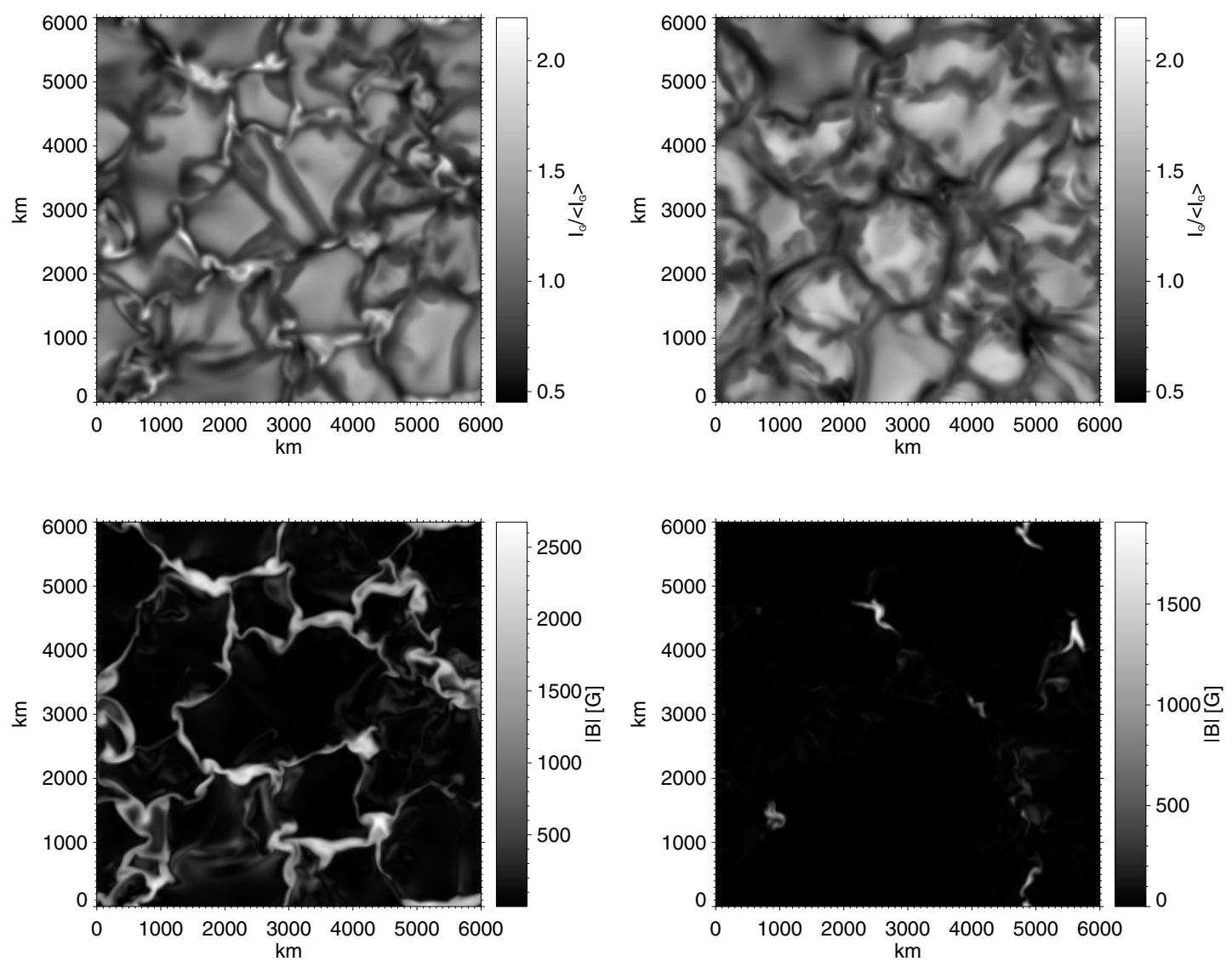

Fig. 3. Relation between normalized $G$-band brightness (upper panels) and magnetic field strength at $\tau=1(500 \mathrm{~nm}$, lower panels) for the snapshot from the 200-G run (left side) and the 10-G run (right side), respectively. While the simulated plage region (200-G run) shows many brightenings of magnetic features exceding the brightness of granules, there are only a few bright features in the simulated quiet region. Note the different grey-scales for the $200-G$ and the $10-G$ results, respectively.
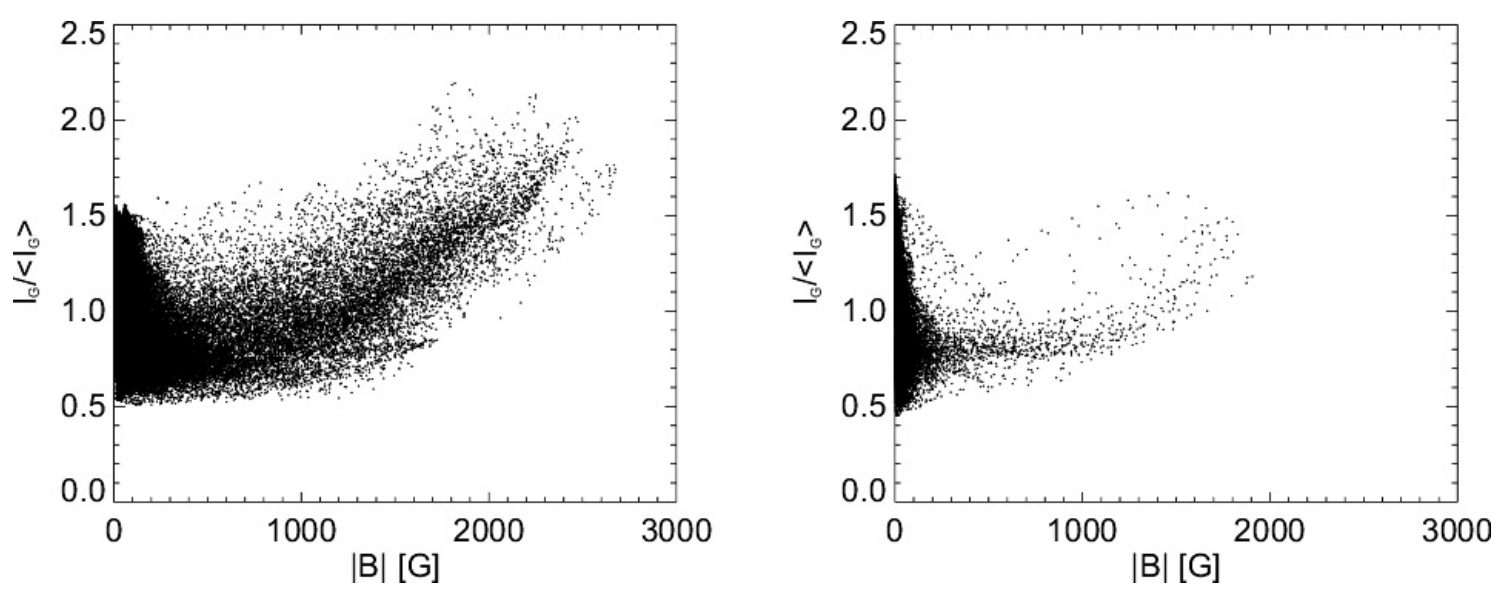

Fig. 4. Scatter plots of $G$-band brightness vs. magnetic field strength (at $\log \tau=-1$ for $500 \mathrm{~nm}$ ) for the 200- $G$ run (left) and for the $10-G$ run $($ right). The brightness of the points with strong magnetic field exceeds that of the (weakly magnetized) granules in the $200-G$ case. The few flux concentrations in the 10-G case are smaller and weaker than the features shown by the 200- $G$ case, and they do not exceed the brightness of the brightest granules.

leaves the flux concentration in the deeper parts of the atmosphere and the radiation thus mainly reflects the properties of the cool intergranular gas surrounding the flux concentrations (the "canopy effect"; see, e.g., Grossmann-Doerth et al. 1988). At small $B$, there is a broad range of $G$-band brightness, which corresponds to bright granules and dark intergranular lanes. In the $10-G$ run, the brightest features are actually associated with granules, where magnetic field is weak. In the image, granules and magnetic elements are easily distinguished by their different sizes and shapes. This information is, of course, lost in the scatter plots. The situation is clearer when we consider only regions harbouring downflowing gas. Almost all (98\%) of the points in downflow regions with $I_{G} /\left\langle I_{G}\right\rangle>1.5$ are associated with strong magnetic field, $|B|>1000 \mathrm{G}$. 

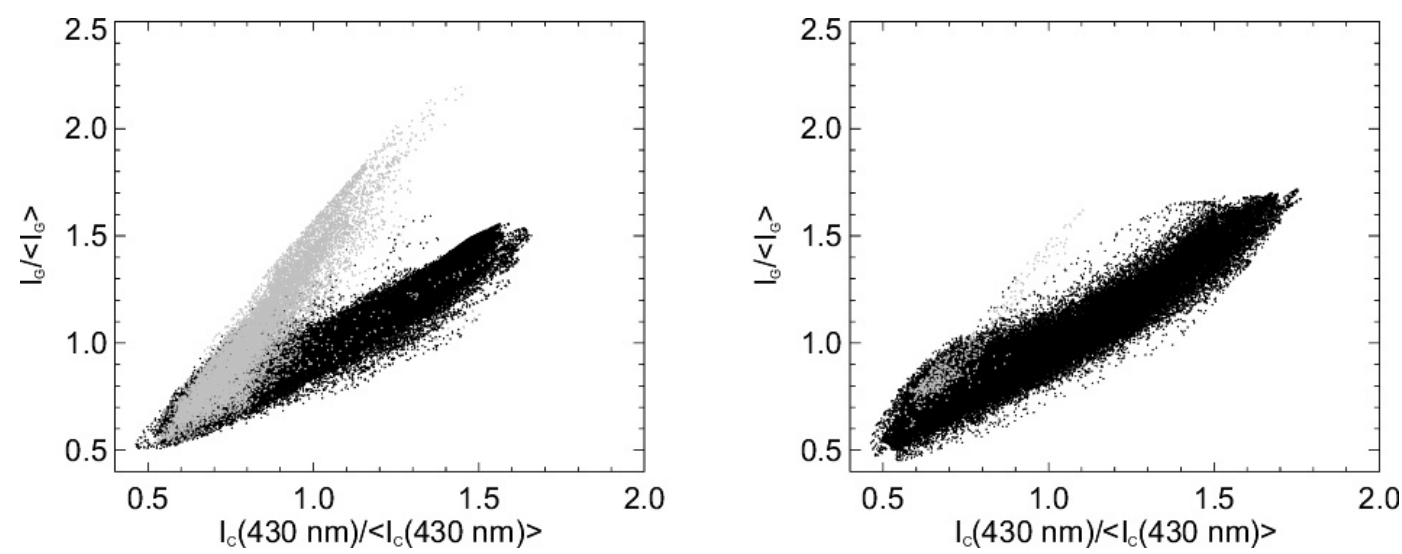

Fig. 5. Scatter plots of $G$-band brightness vs. continuum brightness at $430 \mathrm{~nm}$ for the $200-G$ (left) and $10-G$ (right) runs. The slope of the distributions indicates a clear separation in two components corresponding to magnetic flux concentrations $(|B|>500 \mathrm{G}$, gray dots) and weakly magnetized atmosphere (black dots), mainly corresponding to granules. In the $10-G$ case, the component corresponding to the magnetic flux concentrations is only weak, reflecting their small area fraction.

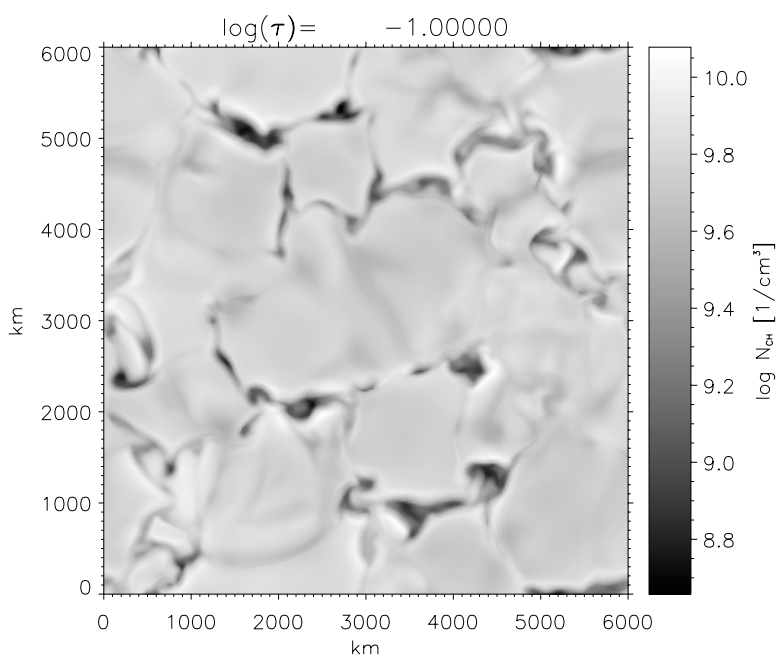

Fig. 6. Number density of $\mathrm{CH}$ molecules at the level $\log \tau=-1.0$ $(500 \mathrm{~nm})$ for the $200-G$ run. The dark regions with low $\mathrm{CH}$ concentration correspond to strong magnetic field concentrations and are cospatial with the brightenings on the $G$-band image (left part of Fig. 3).

Scatter plots of $G$-band brightness versus continuum intensity at $430 \mathrm{~nm}$ for both runs are shown in Fig. 5. The separation of the image pixels in two components with a different slope of $I_{G}$ vs. $I_{C}$ is very similar to the observations of Berger et al. (1998, see their Fig. 3). The component with larger $G$-band intensity for the same continuum intensity (indicated in grey) corresponds to points with strong vertical magnetic field $(|B|>500 \mathrm{G})$, while the other component corresponds to weakly magnetized granules. Figure 5 also shows that although $30 \%-50 \%$ of the strong magnetic fields can be recognized, many points are indistinguishable from points associated with weak fields.

\section{Why are magnetic flux concentrations bright in the $G$ band?}

The $G$-band spectral range is dominated by lines of the $\mathrm{CH}$ molecule and its integrated brightness is thus dependent on

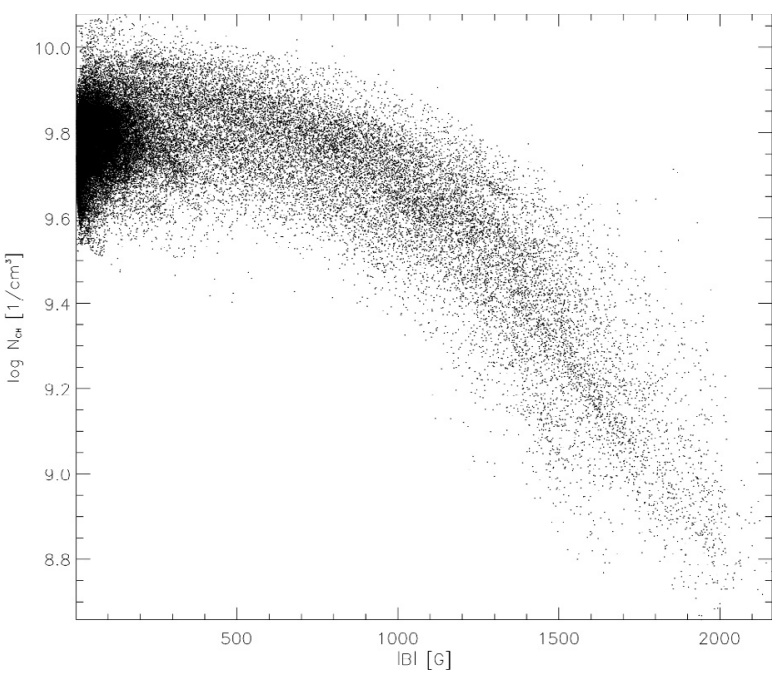

Fig. 7. Scatter plot of the number density of $\mathrm{CH}$ molecules vs. magnetic field strength (both at $\log \tau=-1.0$ for $500 \mathrm{~nm}$ ), calculated for the $200-G$ run.

the strengths of those lines. The mechanism for the enhancement of $G$-band brightness in magnetic flux concentrations can be revealed by studying the dependence of the $\mathrm{CH}$ molecule concentration on the atmospheric parameters within and without magnetic flux concentrations, respectively.

A map of the number density of $\mathrm{CH}$ molecules at the optical depth level $\log \tau=-1$ (at $500 \mathrm{~nm}$ ), which roughly corresponds to the level of $\mathrm{CH}$ line formation, is shown in Fig. 6. For the 200- $G$ case, comparison with the left side of Fig. 3 shows that there is a strong (more than one order of magnitude) decrease of the $\mathrm{CH}$ molecule concentration at the locations where the $G$-band brightness is high and the magnetic field is strong. The clear anticorrelation $(r=-0.85)$ of $\mathrm{CH}$ number density and magnetic field strength for strong magnetic fields $(B>500 \mathrm{G})$ evident from the scatter plot of both quantities shown in Fig. 7 confirms this impression. This figure also indicates the number density depends roughly quadratically on $B$, which fits well to the similar dependence of $G$-band brightness on $B$ as revealed by Fig. 4 . These results suggest that the $G$-band bright points 

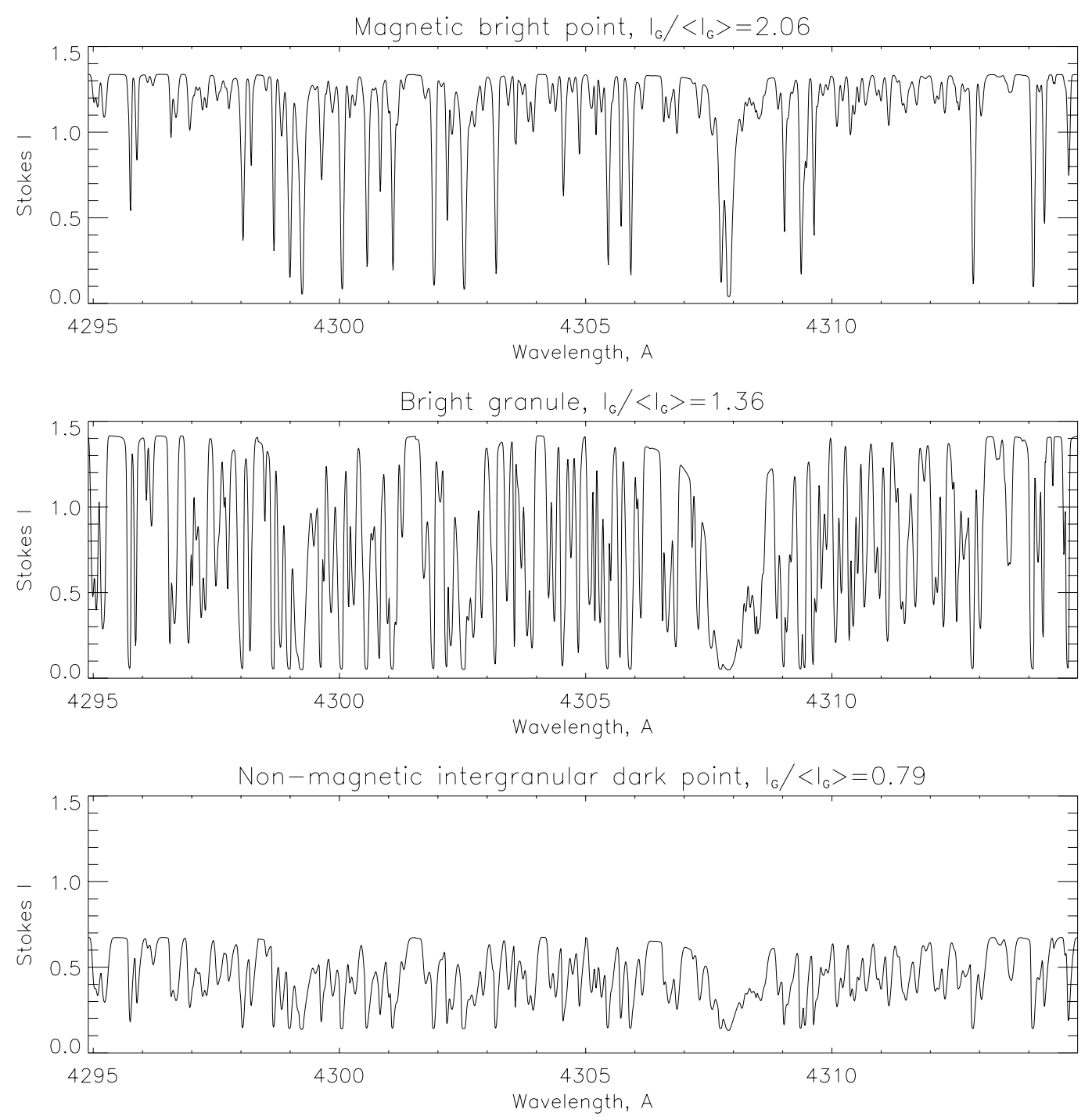

Fig. 8. The Stokes $I$ spectra of a magnetic bright point (top), a bright granule (middle) and a non-magnetic intergranular dark point (bottom). The spectra are normalized to the average continuum intensity at $430 \mathrm{~nm}$. The $\mathrm{CH}$ lines are drastically weakened in the magnetic bright point, the remaining strong lines being atomic lines. Arrows indicate the $\mathrm{CH}$ line at $430.4383 \mathrm{~nm}$, which is used as basis for Fig. 9.

with $B>500 \mathrm{G}$ are caused by the reduced number density of $\mathrm{CH}$ molecules in the magnetic flux concentrations.

Actually, there are two effects that can lead to a local brightness enhancement in $G$ band filtergrams: (1) a larger continuum intensity and (2) weaker $\mathrm{CH}$ lines. Figure 5 shows that the first effect is responsible for the brightening of (non-magnetic) granules: the normalized $G$-band intensity is, on average, equal to the normalized continuum intensity at $430 \mathrm{~nm}$. For the magnetic bright points, the $G$-band intensity is much larger than the normalized continuum intensity, indicating that weakening of the $\mathrm{CH}$ lines is an important factor. This is illustrated by Fig. 8, which shows the $G$-band spectra of a magnetic bright point, a bright granule, and a weakly magnetic intergranular dark point, all normalized to the average continuum intensity at $430 \mathrm{~nm}$. While the granule has a $G$-band intensity enhancement nearly equal to the corresponding normalized continuum intensity, the magnetic bright point (with about the same continuum intensity) exhibits a much larger $G$-band brightness owing to the drastic weakening of the $\mathrm{CH}$ lines. The intergranular dark point has a low $G$-band brightness mainly because of its low continuum intensity.

The large $G$-band intensity of magnetic bright points and the weakening of the $\mathrm{CH}$ lines are connected with the higher temperature (at equal optical depth) and the lower density in the flux concentrations. To a good approximation, the latter are in total (gas + magnetic) pressure balance with their environment leading to an average Wilson depression of about $170 \mathrm{~km}$ (Shelyag et al. 2004). The vertically outgoing radiative flux in the flux concentrations is provided by lateral radiation from the "hot walls" of the flux concentrations, leading to a total radiative cooling rate of the flux concentrations near to zero (Vögler 2003) and a flatter temperature gradient compared to the the non-magnetic solar surface, where a strong radiative cooling is balanced by heating through convection.

The strong weakening of the $\mathrm{CH}$ lines in magnetic flux concentrations can be understood by considering Fig. 9, which shows the main thermodynamic quantities and the number density of $\mathrm{CH}$ molecules for the average atmospheres 

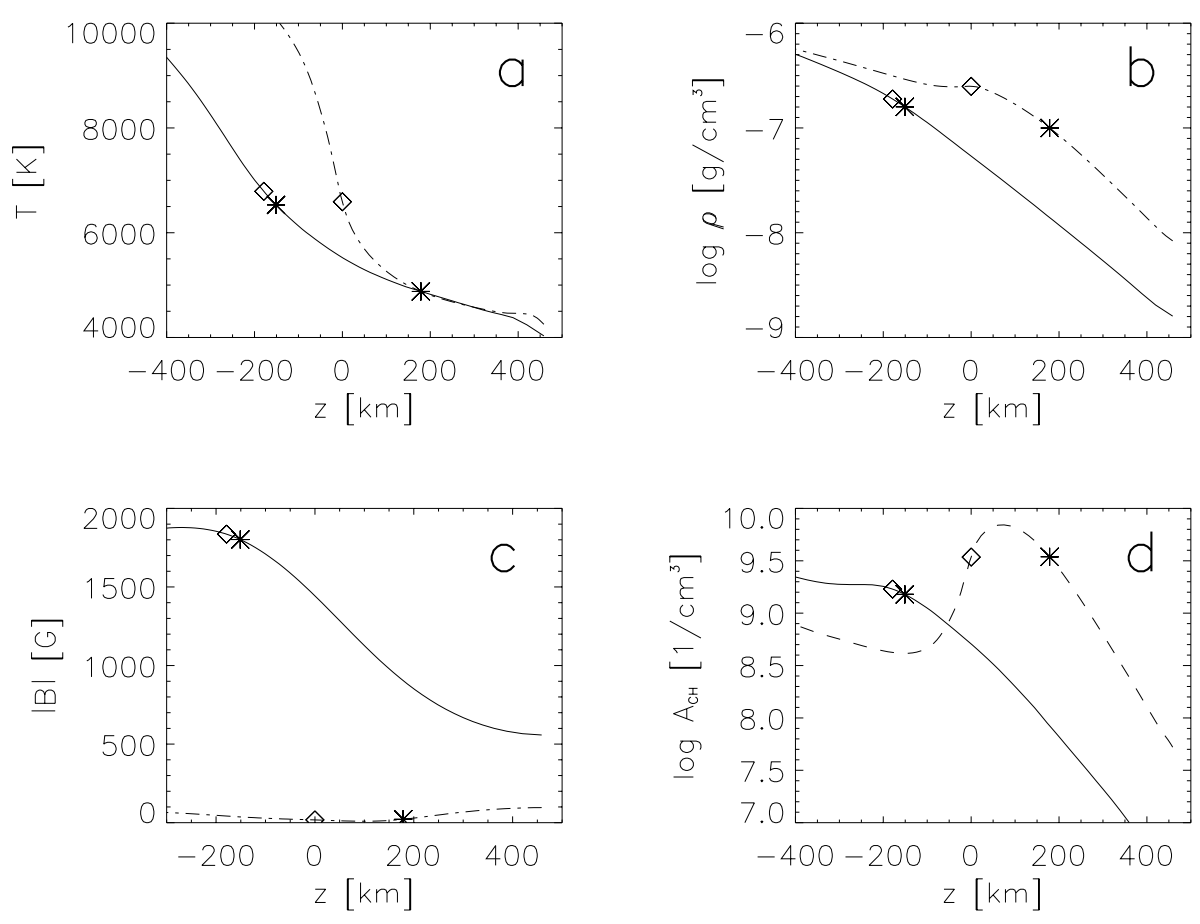

Fig. 9. Temperature a), density b), magnetic field strength c) and number density of $\mathrm{CH}$ molecules d) as functions of geometrical depth for the average weakly magnetized atmosphere (mostly granules, $|B|<50 \mathrm{G}$; dot-dashed lines) and for the average atmosphere corresponding to magnetic bright points $\left(|B|>1000 \mathrm{G}, I_{G} /\left\langle I_{G}\right\rangle>1.56\right.$; solid lines). The diamonds and asterisks on the curves indicate the optical depth levels $\tau=2 / 3$ for the $G$-band continuum at $430 \mathrm{~nm}$ and for the center of the $\mathrm{CH}$ line at $430.4383 \mathrm{~nm}$, respectively. $z=0$ corresponds to the $\tau=2 / 3$ level at the $G$-band continuum for the average weakly magnetized atmosphere. Owing to the lower $\mathrm{CH}$ abundance in the magnetic flux concentrations above $z \approx 0$, the formation region of the $\mathrm{CH}$ lines is compressed in height and shifted geometrically downward to a region with larger temperature and smaller temperature gradient than outside. The resulting combination of larger continuum intensity and strongly weakened $\mathrm{CH}$ lines leads to a brightness enhancement in the $G$-band.

corresponding to the magnetic bright points $(B>1000 \mathrm{G}$ and $I_{G} /\left\langle I_{G}\right\rangle>1.56$; solid curves) and the weakly magnetized part of the atmosphere (mostly granules, $B<50 \mathrm{G}$; dot-dashed curves). Diamond and asterisk symbols indicate the heights at which $\tau=2 / 3$ is reached in $G$-band continuum at $430 \mathrm{~nm}$ and in the center of the $\mathrm{CH}$ line at $430.4383 \mathrm{~nm}$ (taken as a representative for the $\mathrm{CH}$ lines), respectively, and thus roughly cover the height range over which the $\mathrm{CH}$ lines are formed.

The prominent maximum in the $\mathrm{CH}$ number density for the granules (Fig. 9d) is produced by the rapid outward drop in temperature around the continuum-forming layers (Fig. 9a) and the associated density plateau (Fig. 9b). On the other hand, the low density in the magnetic flux concentrations leads to a strong depletion of $\mathrm{CH}$ above $z \approx 0$. Therefore, the optical depth scale at the center of any $\mathrm{CH}$ line is geometrically shifted downward more strongly than the corresponding level for the $G$-band continuum, so that the line is formed over a height range of only $\simeq 30 \mathrm{~km}$ (in comparison to about $180 \mathrm{~km}$ for the weakly magnetic atmosphere). Together with the flatter temperature gradient in the flux concentrations, this leads to a much smaller temperature difference between the layers where the continuum and where the $\mathrm{CH}$ line core is formed as in the case of the non-magnetic atmosphere: the line becomes weaker in regions of strong magnetic field. In combination with their high continuum intensity due to lateral radiative heating of the flux concentrations in that height range, this leads to a strong increase of $G$-band brightness in the magnetic flux concentrations.

\section{Comparison with observational data}

The rms contrast of our synthetic $G$-band images is about $25 \%$ for the $200-G$ run, which is much larger than the value of about $10 \%$ obtained from ground-based observations under very good seeing conditions. Apparently, the image degradation due to the turbulent terrestrial atmosphere leads to reduced values of the contrast observed on the ground. In order to compare our results with observations, we therefore artificially degrade the synthetic $G$-band images, thus mimicking the effect of the atmosphere and telescope. We have convolved our images with a point spread function (PSF) consisting of two parts: an Airy function $A_{\lambda, D}$ for the telescope and a function representing the effect of the atmosphere (Nordlund 1984; Collados \& Vázquez 1987):

$$
\operatorname{PSF}(r)=A_{\lambda, D}(r)+\frac{b}{\left(a^{2}+r^{2}\right)^{3 / 2}} .
$$

Here $\lambda=430 \mathrm{~nm}$ (wavelength of observation) and $D$ is the telescope aperture. The parameters $a$ and $b$ of the atmosphere function describe its width and amplitude, respectively. As long as the atmosphere PSF is much wider than the telescopic PSF, the former does only degrade the rms intensity contrast without strongly affecting the spatial resolution of the image (the latter 

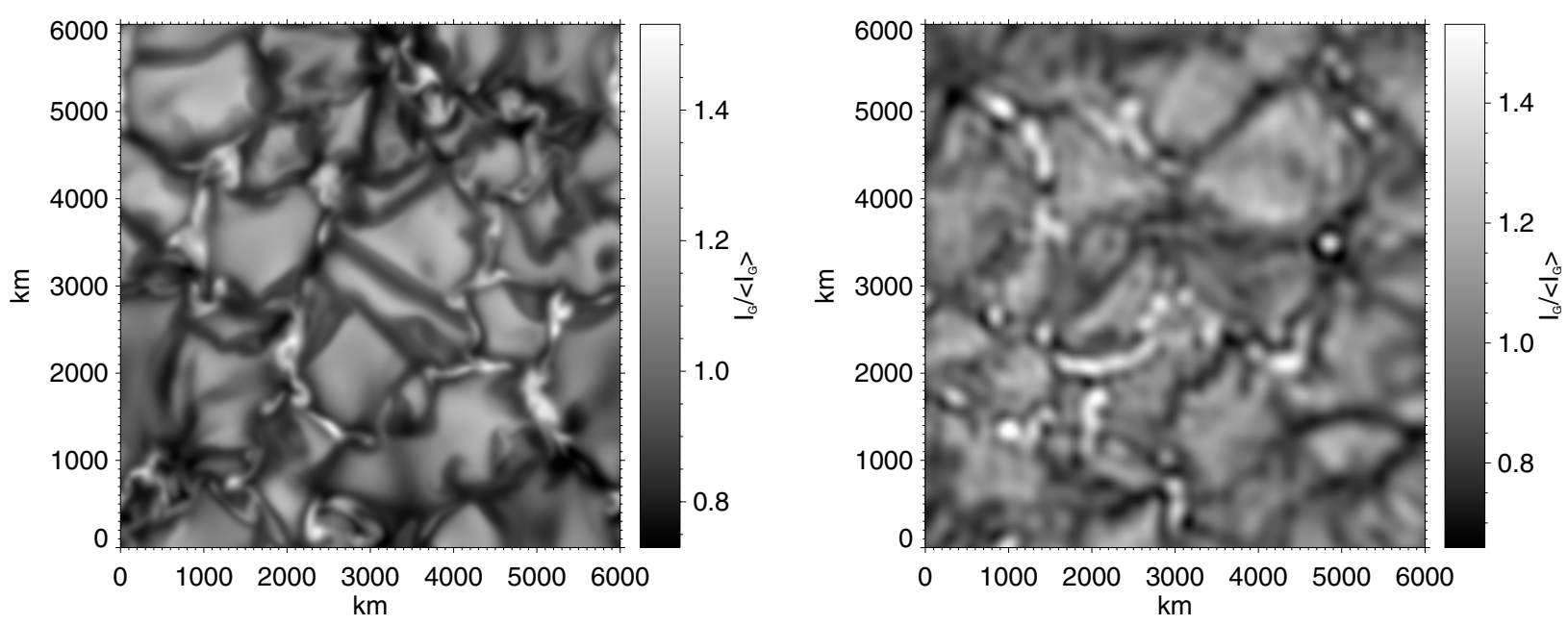

Fig. 10. Simulated and observed $G$-band images. Left: synthetic $G$-band image of the simulated area after spatial smoothing by the function mimicking the difraction by the telescope and the image degradation by the Earth's atmosphere. Right: observed $G$-band image with a similar area fraction of $G$-band bright points as in the 200- $G$ simulation (subfield of an image taken with the Dutch Open Telescope on La Palma, courtesy: P. Sütterlin).

being more affected by speckle effects, which are not included in our simplistic treatment). In this case, the smoothing does not depend sensitively on the precise value of $a$ since the wings of the atmosphere PSF are anyway very broad. Effectively the only remaining free parameter is then $b$, the relative amplitude of the atmosphere PSF. We fix $b$ by requiring that the rms intensity contrast of the smoothed image matches the value for the observed image.

The observed image of an active region, which was used for the comparison, was recorded on May 22, 2002 with the Dutch Open Telescope (DOT) (Hammerschlag et al. 1998) on La Palma (Spain). The selected part of this image has the same physical size as the simulated domain and a similar area fraction of $G$-band bright points as $200-G$ run. Figure 10 shows the smoothed simulated image (left) and the observed image (right). Choosing $120 \mathrm{~km}$ for the $F W H M$ of the Airy function (corresponding to the $45 \mathrm{~cm}$ aperture of the DOT telecope) and a $F W H M$ of $1500 \mathrm{~km}$ for the atmosphere function we find that the smoothed simulated image matches the rms contrast of the observed image of about $7.4 \%$ for $b=0.004$. The degradation of the contrast by a factor 3.4 is comparable to the correction factors obtained by Collados \& Vazquez (1987) assuming a similar form of the PSF. In order to evaluate the consistency of our smoothing procedure we compare in Fig. 11 the $G$-band brightness distribution functions of simulated (dash-dotted line), observed (dashed line), and smoothed simulated (solid line) images. The latter two curves show a very good agreement although no further fitting procedure has been applied. Schüssler et al. (2003) have applied the same procedure to data from the new Swedish Solar Telescope (SST) on La Palma new SST and also obtained matching brightness distribution functions with similar parameter values.

Scatter plots of the continuum intensity at $432 \mathrm{~nm}$ versus $G$-band intensity for the simulated smoothed image (left) and for the observed image (right) are shown in Fig. 12. The two populations of bright points, which we have already identified to represent strongly and weakly magnetized structures,

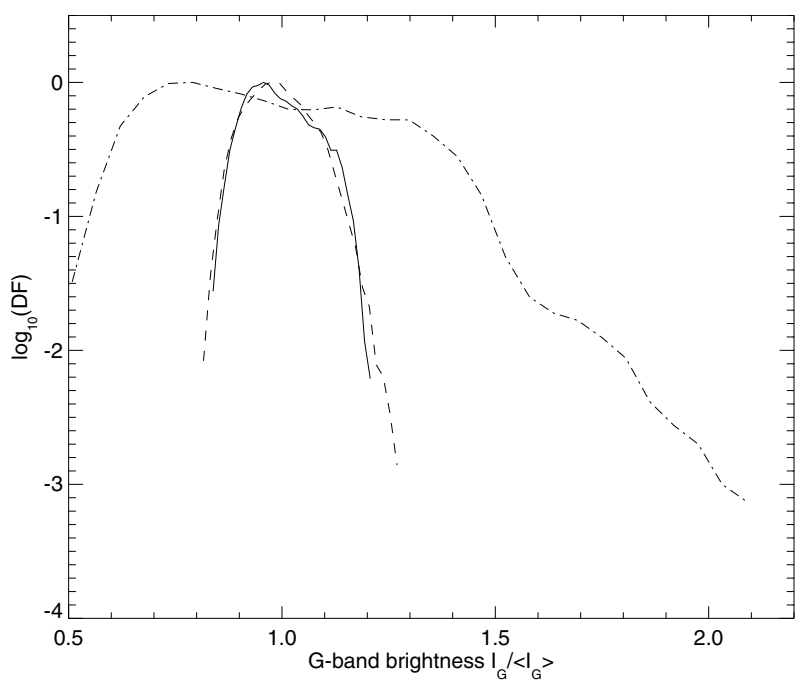

Fig. 11. Distribution functions of $G$-band brightness for the simulated (dash-dotted line), observed (dashed line), and smoothed simulated (solid line) images. The simulated image corresponds to the snapshot from the $200-G$ run.

respectively, are clearly visible (cf. Fig. 5). This confirms the magnetic nature of the intergranular $G$-band brightenings (see also Berger \& Title 1998, 2001).

\section{Conclusions}

Our results are based on realistic 3-D radiative MHD simulations with high horizontal grid resolution $(\sim 20 \mathrm{~km})$ and on a detailed treatment of the $G$-band spectrum synthesis. By comparing simulation results with observations, we have shown that the $G$-band brightening of small magnetic flux concentrations in the solar photosphere is most probably due to the lateral heating and partial evacuation of the magnetic structures. The values of the observed $G$-band contrast are quantitatively reproduced after smoothing the simulated image to mimic the image 

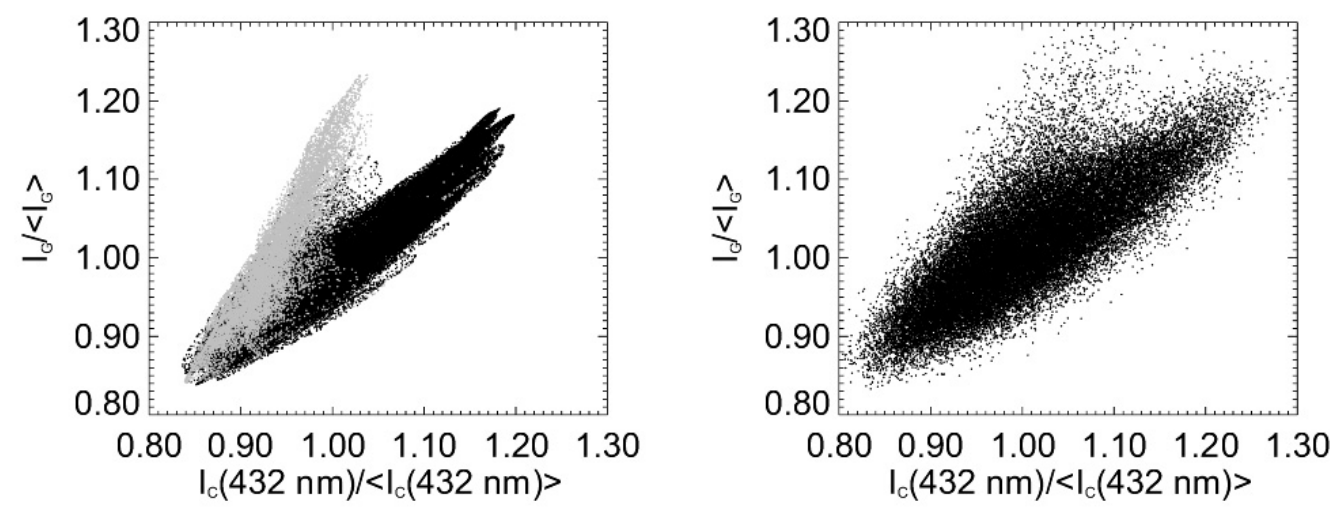

Fig. 12. Scatter plots of normalized $G$-band versus continuum $(432 \mathrm{~nm})$ intensity for the synthetic (200-G run, left) and observed (right) images. The plots show the separation in two components for both observed and simulated images. Grey dots on the plot for the simulated image correspond to the magnetic component $(B>500 \mathrm{G})$.

deterioration by telescope and atmosphere. There is a clear spatial correlation of the $G$-band brightenings and magnetic flux concentrations. These brightenings are caused by a strong reduction in the number density of the $\mathrm{CH}$ molecule in the magnetic flux concentrations, leading to a downward shift of the $G$ band optical depth scale and thus to weakening of the $\mathrm{CH}$ lines in the $G$-band. Granules appear bright in the $G$-band only because their high continuum intensity. Very good agreement between the results of the simulations and the brightness distribution functions of the observed images confirms the physical model of magnetic flux concentrations as laterally heated and evacuated structures.

Acknowledgements. We thank R. Rutten for clarifying discussion about the physical origin of the $G$-band brightenings. The data from the Dutch Open Telescope have been kindly provided by Peter Sütterlin. S. V. Berdyugina acknowledges support by the Academy of Finland, grant 4309.

\section{References}

Berdyugina, S. V., Solanki, S. K., \& Frutiger, C. 2003, A\&A, 412, 513 Berdyugina, S. V., \& Solanki, S. K. 2002, A\&A, 385, 701

Berger, T. E., \& Title, A. M. 1996, ApJ, 463, 365

Berger, T. E., \& Title, A. M. 2001, ApJ, 553, 449

Berger, T. E., Schrijver, C. J., Shine, R. A., et al. 1995, ApJ, 454, 531

Berger, T. E., Loefdahl, M. G., Shine, R. S., \& Title, A. M. 1998, ApJ, 495, 973

Collados, M., \& Vázquez, M. 1987, A\&A, 180, 223

Deinzer, W., Hensler, G., Schüssler, M., \& Weisshaar, E. 1984, A\&A, 139,426

Delbouille, L., Roland, G., \& Neven, L. 1973, Photometric Atlas of the Solar Spectrum (Liège: Institut d'Astrophysique de L'Université de Liège)

De Pontieu, B. 2002, ApJ, 569, 474

Fligge, M., Solanki, S. K., \& Unruh, Y. C. 2000, A\&A, 353, 380

Gandorfer, A. 2002, The Second Solar Spectrum, Vol. 2: $3910 \AA$ to $4630 \AA$ (Zürich: vdf), ISBN 3728128554

Grossmann-Doerth, U., Schüssler, M., \& Solanki, S. K. 1988, A\&A, 206, L37

Hammerschlag, R. H., Bettonvil, F. C. M. 1998, New Astron. Rev., 42, 485
Knölker, M., Grossmann-Doerth, U., Schüssler, M., \& Weisshaar, E. 1991, Adv. Space Res., 11, 285

Krivova, N. A., Solanki, S. K., Fligge, M., \& Unruh, Y. C. 2003, A\&A, 399, L1

Langhans, K., Schmidt, W., \& Tritschler, A. 2002, A\&A, 394, 1069

Muller, R. 1985, Sol. Phys., 100, 237

Muller, R., \& Roudier, T. 1984, Sol. Phys., 94, 33

Nordlund, A. 1984, in Small-Scale Dynamical Processes in Quiet Stellar Atmospheres, ed. S. L. Keil, Sunspot/NM: Sacramento Peak Observatory, 174

Parker, E. N. 1983, ApJ, 264, 635

Rutten, R. J., Hammerschlag, R. H., Sütterlin, P., \& Bettonvil, F. C. M. 2001, in Advanced Solar Polarimetry - Theory, Observation, and Instrumentation, ed. M. Sigwarth (San Francisco: Astron. Society of the Pacific), ASP Conf. Ser., 236, 25

Sánchez Almeida, J., Asensio Ramos, A., Trujillo Bueno, J., \& Cernicharo, J. 2001, ApJ, 555, 978

Schrijver, C. J., Title, A. M., Harvey, K. L., et al. 1998, Nature, 394, 152

Schüssler, M. 2003, in Third International Workshop on Solar Polarization, ed. J. Trujillo Bueno, \& J. Sanchez Almeida, ASP Conf. Ser., in press

Schüssler, M., Shelyag, S., Berdyugina, S., Vögler, A., \& Solanki, S. K. 2003, ApJ, 597, L173

Shelyag, S., Solanki, S., Vögler, A., \& Schüssler, M. 2004, in preparation

Solanki, S. K. 1993, Space Sci. Rev., 63(1/2), 1

Spruit, H. C. 1976, Sol. Phys., 50, 269

Spruit, H. 2000, Space Sci. Rev., 94, 113

Spruit, H., Schüssler, M., \& Solanki, S. 1991, in Solar Interior and atmosphere, ed. M. Mattews, \& M. Giampapa (Tucson, AZ: University of Arizona Press), 890

van Ballegooijen, A. A., Nisenson, P., Noyes, R. W., et al. 1998, ApJ, 509,435

Vögler, A., \& Schüssler, M. 2003, Astron. Nachr./AN, 324, 399

Vögler, A., Shelyag, S., Schüssler, M., et al. 2004, in preparation

Vögler, A. 2003, Ph.D. Thesis, University of Göttingen, Germany, http://www.linmpi.mpg.de/ msch/Thesis_Voegler.pdf

Vögler, A., Shelyag, S., Schüssler, M., et al. 2003, in Modelling of Stellar Atmospheres, ed. N. E. Piskunov, W. W. Weiss, \& D. F. Gray (San Francisco: Astron. Soc. of the Pacific), IAU-Symp., 210,157 